\title{
Editorial
}

Nephrology

\section{Would Not Be Surprised. What Next?}

\author{
Laura P. Perry Kenneth E. Covinsky \\ Division of Geriatrics, University of California at San Francisco, San Francisco, CA, USA
}

Ramer [1] asks of nephrologists a question gaining traction as an efficient to estimate prognosis: "Would you be surprised if this patient died in the next 12 months?" They found a strong relationship between the answer and the likelihood of subsequent hospitalization. The authors describe the "surprise question" as being based on "intuition." Yet, we believe that it reflects far more than a gut feeling, but the sum total knowledge that develops from a close relationship with a patient. It reflects what a patient looks like while walking from the waiting room to the examination room, or getting from a chair to the examination table. It reflects knowledge of the patient's home situation as well as their cognitive and psychological state. All of these characteristics that a clinician might observe when they know a patient well are very powerful determinants of prognosis. Many of these are measurable and can predict outcomes ranging from utilization to mortality.

The deep knowledge of a patient that guides prognostication can serve as guide that helps a clinician prevent clinical decline and improve quality of life. The authors aptly note that the provider intuition that underlies the surprise question should be employed for the benefit of the patient. They include 2 excellent suggestions: applying prognostic information to shared decision-making and the prioritization of high-risk patients for closer attention to prevent hospitalization. Yet, we believe the recognition that a patient has a high risk of mortality should also lead to a shift in the overall plan of care. Measures that prolong survival should be balanced with and complemented by those that promote a high quality of life. We encourage nephrologists not to think of this palliative care focus as an on-off switch, but rather a spectrum of how care is delivered. Often, the focus on length and quality of life will be fully compatible.
Over time, the amount the relative effort focused on each goal may shift.

Both of these goals can be met by explicitly focusing on key domains of functioning, including cognitive, physical, and psychological components. These components of functioning are often the same parameters that underlie a clinician's answer to the surprise question. Addressing them commonly reduces the need for hospitalization. Care focused on patient function demands multidisciplinary care, a model of care that lends itself well to nephrology. When a nephrologist cares for a patient with a limited prognosis, we encourage them to think through issues within the domains of cognitive, physical, and psychological function to develop care plans and goals that will improve quality of life. In many cases, simply knowing whom to ask for consultation will go a long way toward helping your patient.

\section{Cognitive Function}

Cognitive impairment is associated with a substantial increase in risk of near-term mortality. It is extremely common in adults with late-stage kidney disease, and often under-recognized. Up to $70 \%$ of hemodialysis patients over age 55 years exhibit moderate-severe cognitive impairment [2]. Identification of cognitive impairment is critical to determining how care should be delivered. For example, it markedly affects a patient's ability to adhere to a complex medication and treatment regimen and changes the calculation of how decisions about care are made and by whom.

It is no surprise that the vascular changes that affect nephrons also affect neurons. Vascular dementia is a very

\section{KARGER}

(C) 2020 S. Karger AG, Basel 
common comorbidity, and marked not primarily by memory loss but by executive dysfunction - difficulty with decision-making and organization. Nephrologists may be the first to identify these deficits in patients who "keep changing their mind about dialysis." If you find yourself wondering why a previously adherent patient can no longer manage their medications well, consider a brief cognitive screen such as the Mini-Cog [3]. Even better, incorporate cognitive screening as a routine part of care in high-risk patients. If screening tests are abnormal, referral to an expert such as a neurologist, geriatrician, psychiatrist, or neuropsychologist is warranted. In patients with known cognitive impairment, an occupational therapist can often help with strategies for managing day-to-day tasks and appropriate adaptive equipment. Many occupational therapists and speech-language pathologists are trained in cognitive evaluation and rehabilitation.

\section{Physical Function and Frailty}

Recognizing that a patient's death would not be surprising should also lead to a recognition that decline in physical function is likely. The last 2 years of life are associated with an increased incidence of disability in one or more activities of daily living [4], and advancing kidney disease often goes hand-in-hand with functional decline [5]. For the sarcopenia, weakness and falls [6] that frequently accompany late-stage $\mathrm{CKD}$, physical therapists are invaluable. We recommend referral early and often. Occupational therapists can assess needs and are particularly helpful in recommending appropriate adaptive equipment. As patients become more disabled, they may have unmet caregiving needs or require assistance with transportation in order to make it to appointments. Anticipating these concerns and referring to a team social worker makes it possible to consider adaptive interventions such as obtaining home health aides.
Nephrologists have extensive experience in recognizing the harms of medications in their patients, and dose-adjusting or discontinuing accordingly. Frail adults getting closer to the end of life are more susceptible to the harms of medications that cross the blood-brain barrier, even when appropriately dose-adjusted. Dizziness, drowsiness, dry mouth, and constipation are common quality-of-lifethreatening side effects. Deprescribing can often immensely relieve these symptoms. We recommend a medication review to identify potential culprits [7]. A knowledgeable pharmacist or geriatrician can assist with this process.

\section{Psychological/Emotional Well-Being}

Pharmacologic therapies have a role in improving quality of life, but in patients with late-stage kidney disease, they are unlikely to succeed as a sole treatment for the many challenges of aging, being ill, and nearing death. Patients may have difficulty sleeping, fear of dying, or simply difficulty coping with serious illness. Referrals to chaplains, psychologists, or therapists are frequently warranted.

As disease progresses and function declines, the role of the physician evolves from disease prevention to relief of suffering. Many late-life patients will be well known to their nephrologist. Sometimes, patients need their nephrologist to serve as a trusted confidant, helping them manage the quality-of-life concerns that accumulate toward the end of life. If your intuition informs you that a patient's death would not come as a surprise, allow that same intuition to steer you toward the type of doctoring that the patient needs most in the precious time left.

\section{Disclosure Statement}

There is no funding or other conflict of interest relevant to this paper.

\section{References}

1 Ramer SJ, Baddour NA, Siew ED, Salat H, Bian A, Stewart TG, et al. Nephrology provider surprise question response and hospitalizations in older adults with advanced CKD. Am J Nephrol. DOI: 10.1159/000509046.

2 Bugnicourt JM, Godefroy O, Chillon JM, Choukroun G, Massy ZA. Cognitive disorders and dementia in CKD: the neglected kidney-brain axis. J Am Soc Nephrol. 2013;24(3): 353-63.
3 Available from: https://mini-cog.com. Accessed 2020 May 3.

4 Smith AK, Walter LC, Miao Y, Boscardin WJ, Covinsky KE. Disability during the last two years of life. JAMA Intern Med. 2013;173(16): 1506-13.

5 Kurella Tamura M, Covinsky KE, Chertow GM, Yaffe K, Landefeld CS, McCulloch CE. Functional status of elderly adults before and after initiation of dialysis. N Engl J Med. 2009; 361(16):1539-47.
6 Hall RK, Sloane R, Pieper C, Van Houtven C, LaFleur J, Adler R, et al. Competing risks of fracture and death in older adults with chronic kidney disease. J Am Geriatr Soc. 2018; 66(3):532-8.

7 Triantafylidis LK, Hawley CE, Perry LP, Paik JM. The role of deprescribing in older adults with chronic kidney disease. Drugs Aging. 2018 11;35(11):973-84. 